Research Article

\title{
Effects of gestational exercise on hyperoxia-induced brain damage in the newborn
}

\author{
Mustafa Dilek $^{1}$, Gokce Kaya Dincel ${ }^{1}$, Ayhan Cetinkaya ${ }^{2}$, Gamze Dilek ${ }^{3}$, Mervan Bekdas ${ }^{1}$, Erol \\ Ayaz $^{2}$, Nimet Kabakus ${ }^{1}$ \\ ${ }^{I}$ Bolu Abant Izzet Baysal University, Faculty of Medicine, Department of Pediatrics, Bolu, Turkey \\ ${ }^{2}$ Bolu Abant Izzet Baysal University, Experimental Animal Application and Research Center, Bolu, Turkey \\ ${ }^{3}$ Abant Izzet Baysal University, Izzet Baysal Physical Therapy and Rehabilitation Training and Research Hospital, \\ Bolu, Turkey
}

\section{ABSTRACT}

Aim: Preterm infants encounter hyperoxia relatively early on as they leave the intrauterine environment earlier than expected, while also being exposed to a higher level of hyperoxic stress due to insufficiencies in their antioxidant defense mechanisms. With that in mind, we investigate whether running exercises performed during pregnancy can contribute to the development of tolerance to neonatal hyperoxic brain damage.

Method: While two female rats maintained a sedentary pregnancy, one female rat performed the mandatory running exercise for 30 minutes for five days a week throughout the pregnancy. Following delivery, the sedentary rats and the exercised rat were kept together with their offspring for five days at oxygen concentrations above 80 percent in order to induce brain damage. The offspring were sacrificed on postnatal Day 7 and brain/body ratio measurements were obtained.

Results: The brain/body ratios in the control, hyperoxia and exercise-hyperoxia groups were found to be median (IQR) 0.074(0.68-0.77), 0.065(0.06-0.067) and 0.064(0.060-0.068), respectively. The brain/body ratios of the offspring of the mothers in the hyperoxia group were found to be significantly lower than the control group ( $p=0.002)$, irrespective of exercise $(p=0.007)$. No statistically significant difference was noted between the offspring of the sedentary and the exercised mothers in the hyperoxia group $(p=0.94)$.

Conclusion: Hyperoxia was found to result in lower brain mass relative to total body mass. This finding, which indicates the presence of microcephaly, reflects the negative effects of hyperoxia on brain development. Contrary to expectations, exercises performed during pregnancy had no significant effect on the brain/body weight ratio of the offspring.

Keywords: Gestational exercise; cognitive functions; hyperoxic brain injury; neonatal; rat model; offspring. 
Corresponding Author: Dr. Mustafa Dilek,

Department of Pediatrics, Bolu Abant Izzet Baysal University Faculty of Medicine, 14280, Gölköy, Bolu, Turkey

E-mail: mustafadilek@gmail.com

ORCID ID: https://orcid.org/0000-0002-3802-0336

Received 2018-07-31, Revisions 2018-09-04

Accepted 2018-09-06

Publication Date 2018-10-01

\section{Introduction}

Preterm infants encounter hyperoxia relatively early on as they leave the intrauterine environment earlier than expected, and are also exposed to a higher level of hyperoxic stress due to the insufficiencies of their antioxidant defense mechanisms. Both experimental and clinical trials have demonstrated the toxic effects of hyperoxia on the brain. Exposure to high concentrations of oxygen has been identified as the main in the pathogenesis of bronchopulmonary dysplasia and premature retinopathy, which are the main causes of longterm morbidity in preterm infants [1]. Exercising throughout pregnancy can have beneficial effects on the health of both the mother and infant [2]. Exercising during pregnancy is known to have positive effects on antioxidant capacity and neuronal development, while its potential influence on brain damage in preterm infants exposed to oxidative stress is currently unknown. In the present study, we investigate the effects of exercise on hyperoxia-induced brain damage.

\section{Methods}

The study was approved by the AIBU Animal Research Local Ethics Committee and was carried out in AIBU Experimental Animals Application and Research Center. Female Wistar rats were fed with standard pellets and water and were grown in standard cages at room temperature and 50-60 percent humidity under a 12-hour light /dark cycle.

A female rat that was eligible for the running exercise was selected from among the nulliparous rats. The rats were forced to exercise for one week before inclusion, and then up until the time of delivery. Exercises were performed for a total of 30 minutes, five days per week, including running at 3 meters/minute for five minutes, 5 meters/min for five minutes, and 8 meters/min for 20 minutes [3]. The rats thus became accustomed to running on a treadmill. The other two female rats were kept in normal cages.

Consistent with the literature, hyperoxia was induced by exposing the rats to oxygen in an incubator after birth until postnatal day 5 . Oxygen flow was adjusted to keep oxygen concentrations in the incubator at 80-85\%, with a flow rate of $4 \mathrm{~L} /$ hour. Humidity was maintained above $80 \%$. During exposure, oxygen concentrations were measured at least twice a day and were maintained at a constant rate [4-6].

The offspring of one of the mothers provided with normal care in sedentary cages constituted the control group, while the offspring of the other mother was exposed to hyperoxia after birth and constituted the hyperoxia group. The offspring of the mother that exercised was exposed to hyperoxia and constituted the exercised mother offspring group.

The offspring were weighed on postnatal day 5 and then sacrificed under ether anesthesia. The brains were extracted by a craniotomy and weighed, and the brain/body weight ratio was calculated. Mann-Whitney $U$ test was compared with two groups. The data were analyzed using SPSS 18.0 statistical analysis software. 


\section{Results}

The brain/body ratios in the control $(n=9)$, hyperoxia $(n=7)$ and exercise-hyperoxia $(n=7)$ groups were found to be median (IQR) $0.074(0.68-0.77), \quad 0.065(0.06-0.067) \quad$ and 0.064(0.060-0.068), respectively. The brain/body ratios of the offspring of the mothers in the hyperoxia group were found to be significantly lower than the control group $(p=0.002)$, irrespective of exercise $(p=0.007)$. No statistically significant difference was noted between the offspring of the sedentary and exercised mothers in the hyperoxia group $(p=0.94)$ (Figure 1).

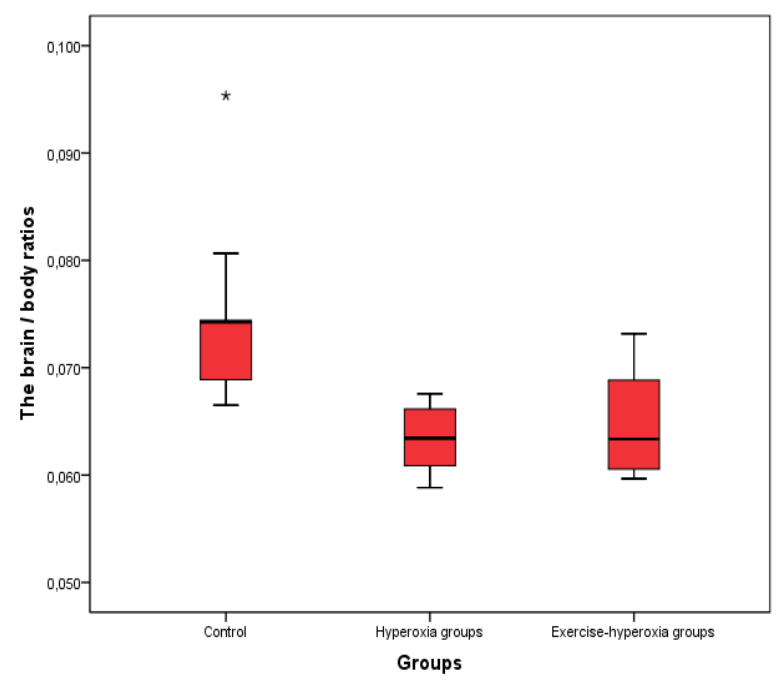

Figure 1. The brain/body ratios in the groups.

\section{Discussion}

Recent studies have shown that increased oxidative stress and a decreased activation of the neurotrophin pathways after the exposure of immature mice to hyperoxia resulted in extensive apoptosis in various regions of the brain [7, 8]. Oxygen triggers inflammation, resulting in the production of various cytokines in the body $[9,10]$, and leading consequently to neurodegeneration [11]. The results of the present study showed that the brain/body weight ratio was lower than in the control group, both in offspring of the exercised mothers exposed to hyperoxia and the offspring of the sedentary mothers exposed to hyperoxia. This indicates that hyperoxia reduces brain mass, or slows its development. Both aerobic and resistance exercises performed during the second trimester of pregnancy were shown to increase the levels of eNOS and NO and to decrease free oxygen radicals in the placenta [12]. Recent studies have demonstrated that swimming exercises performed by mothers during pregnancy can improve the memory function of their offspring, while also increasing hippocampal neurogenesis and neurotrophic factors [13]. Swimming exercises during pregnancy have also been associated with increased antioxidant levels in the brain tissue of the offspring, and mitochondrial mass was reported to be higher in the exercised group [2, 14]. Running exercises performed during pregnancy were shown to increase the levels of BDNF and VEGF in the brain tissue of the offspring, and the exercised group had more successful results in behavioral tests, such as open space and locomotor [3, 15-18]. It was considered in the present study that through all of these mechanisms, exercise during pregnancy may have a positive effect on brain development, in addition to showing protective effects in terms of brain damage caused by hyperoxia. That said, we were unable to find any significant difference between the brain/body weight ratios of the offspring of the sedentary and exercised mothers.

Hyperoxia was found to cause lower brain mass relative to total body mass. This finding, which indicates the presence of microcephaly, indicates also the negative effects of hyperoxia on brain development. Contrary to the expectations, exercises performed during pregnancy had no significant effect on the brain/body weight ratio. There is an apparent 
need for additional studies evaluating the effects of exercise performed during pregnancy on postnatal hyperoxia exposure through different measurement methods.

\section{Compliance with ethical statements \\ Conflicts of Interest: None.}

\section{Acknowledgments}

(This study was supported by Bolu Abant Izzet Baysal University - Scientific Research Projects Coordination Unit with project number 2015.08.23.968. The supplies and inventories of project 2015.08.23.967 were used.)

\section{References}

[1]Saugstad OD. Is oxygen more toxic than currently believed? Pediatrics 2001; 108(5):1203-1205.

[2]Marcelino TB, Longoni A, Kudo KY, Stone $\mathrm{V}$, Rech A, de Assis AM et al. Evidences that maternal swimming exercise improves antioxidant defenses and induces mitochondrial biogenesis in the brain of young Wistar rats. Neuroscience 2013; 246:28-39.

[3] Kim H, Lee SH, Kim SS, Yoo JH, Kim CJ. The influence of maternal treadmill running during pregnancy on short-term memory and hippocampal cell survival in rat pups. International journal of developmental neuroscience : the official journal of the International Society for Developmental Neuroscience 2007; 25(4):243-249.

[4]Yis U, Kurul SH, Kumral A, Cilaker S, Tugyan K, Genc S et al. Hyperoxic exposure leads to cell death in the developing brain. Brain \& development 2008; 30(9):556-562.

[5]Yis U, Kurul SH, Kumral A, Tugyan K, Cilaker S, Yilmaz $\mathrm{O}$ et al. Effect of erythropoietin on oxygen-induced brain injury in the newborn rat. Neuroscience letters 2008; 448(3):245-249.

[6]Tuzun F, Kumral A, Ozbal S, Dilek M, Tugyan K, Duman N et al. Maternal prenatal omega-3 fatty acid supplementation attenuates hyperoxiainduced apoptosis in the developing rat brain. International journal of developmental neuroscience : the official journal of the International Society for Developmental Neuroscience 2012; 30(4):315-323.

[7]Hoehn T, Felderhoff-Mueser U, Maschewski K, Stadelmann C, Sifringer M, Bittigau $\mathrm{P}$ et al. Hyperoxia causes inducible nitric oxide synthase-mediated cellular damage to the immature rat brain. Pediatric research 2003; 54(2):179-184.

[8]Felderhoff-Mueser U, Bittigau P, Sifringer M, Jarosz B, Korobowicz E, Mahler L et al. Oxygen causes cell death in the developing brain. Neurobiology of disease 2004; 17(2):273-282.

[9]Rozycki HJ, Comber PG, Huff TF. Cytokines and oxygen radicals after hyperoxia in preterm and term alveolar macrophages. American journal of physiology Lung cellular and molecular physiology 2002; 282(6):L1222-1228.

[10]Pierce BT, Napolitano PG, Pierce LM, Apodaca CC, Hume RF, Jr., Calhoun BC. The effects of hypoxia and hyperoxia on fetal-placental vascular tone and inflammatory cytokine production. American journal of obstetrics and gynecology 2001; 185(5):1068-1072.

[11] Allan SM, Rothwell NJ. Cytokines and acute neurodegeneration. Nature reviews Neuroscience 2001; 2(10):734-744.

[12]Ramírez-Vélez R, Bustamante J, Czerniczyniec A, Aguilar de Plata AC, 
Lores-Arnaiz S. Effect of Exercise Training on Enos Expression, NO Production and Oxygen Metabolism in Human Placenta. PLoS ONE 2013; 8(11).

[13]Lee H-H, Kim H, Lee J-W, Kim Y-S, Yang $\mathrm{H}-\mathrm{Y}$, Chang H-K et al. Maternal swimming during pregnancy enhances short-term memory and neurogenesis in the hippocampus of rat pups. Brain \& development 2006; 28(3):147-154.

[14]Park J-W, Kim M-H, Eo S-J, Lee E-H, Kang J-S, Chang $\mathrm{H}-\mathrm{K}$ et al. Maternal exercise during pregnancy affects mitochondrial enzymatic activity and biogenesis in offspring brain. Int J Neurosci 2013; 123(4):253-264.

[15] Aksu I, Baykara B, Ozbal S, Cetin F, Sisman AR, Dayi A et al. Maternal treadmill exercise during pregnancy decreases anxiety and increases prefrontal cortex VEGF and BDNF levels of rat pups in early and late periods of life. Neuroscience letters 2012; 516(2):221-225.

[16] M Akhavan M, Miladi-Gorji H, EmamiAbarghoie M, Safari M, SadighiMoghaddam B, A Vafaei A et al. Maternal Voluntary Exercise during Pregnancy Enhances the Spatial Learning Acquisition but not the Retention of Memory in Rat Pups via a TrkB-mediated Mechanism: The Role of Hippocampal BDNF Expression. Iran J Basic Med Sci 2013; 16(9):955-961.

[17] Yin MM, Wang W, Sun J, Liu S, Liu XL, Niu YM et al. Paternal treadmill exercise enhances spatial learning and memory related to hippocampus among male offspring. Behav Brain Res 2013; 253:297304.

[18] Parnpiansil P, Jutapakdeegul N, Chentanez T, Kotchabhakdi N. Exercise during pregnancy increases hippocampal brainderived neurotrophic factor mRNA expression and spatial learning in neonatal rat pup. Neuroscience letters 2003; 352(1):45-48. 